\title{
Is the Cosmological Particle Production Homogeneous?
}

\author{
M. de Campos \\ Departamento de Física, Universidade Federal de Roraima, \\ Av. Ene Garcez 2413, Campus do Paricarãna, Bloco III, \\ Bairro Aeroporto, 69304-000, Boa Vista, Roraima, Brazil
}

(Received on 14 October, 2005)

\begin{abstract}
Using a gauge-invariant formalism we find the density contrast equation in a cosmological scenario with particle production at the expenses of the gravitational field (open system cosmology). First, we find the modes for the density contrast considering that the particle production process participate of the inhomogeneities formation, and in a second phase the creation process mimics the inclusion of the a smooth $\Lambda$ term, in the sense that it is not affects small deviations from homogeneity. The cosmic background has an accelerated regime, where an additional pressure due to the creation process is responsible for the cosmic acceleration. We study in this work if the creation process of particles in the cosmic fluid contributes to the inhomogeneities formation in an accelerated universe.
\end{abstract}

\section{INTRODUCTION}

The mathematical theory of perturbations in homogeneous, isotropic models has been worked over many times in the literature [1]-[7]. Nevertheless, troubling questions still remain about the physical interpretation of density perturbations at early times when the perturbation is larger than the particle horizon, which will here mean when the time for light to travel a characteristic wavelength of the perturbation is larger than the instantaneous expansion time. These questions are particularly relevant to attempt to explain the origin of perturbations which eventually give rise to the structure formation at large scale.

The study of perturbations of an expanding universe would appear to be hopelessly gauge dependent. However, J. Bardeen [2] gave an important contribution to define perturbations that are gauge invariant quantities which were nongeometrical defined with respect to a particular chart. Ellis and Bruni [3] gave an alternative approach to gauge invariant cosmological perturbations with basic variables more closely related to the physical quantities. They look for variables which vanish in the background, since the quantities of this kind are always gauge independents. The gauge invariant key variable is the comoving fractional spatial gradient of the energy density. In this study, we use an approach to cosmological perturbations which is thermodynamically oriented, gauge-invariant description of scalar cosmological perturbations around a flat homogeneous universe. All basic variables have their meaning on 'comoving hyper-surfaces', orthogonal to the matter world lines. Thermodynamic perturbations relations in Minkowski spacetime turn-out to be valid in the expanding universe, provided all perturbed quantities are replaced by their gauge-invariant counterparts. We consider a background with a cosmic creation of particles at the expenses of the gravitational field. The creation process redefines the energy momentum tensor and an additional pressure appear, the pressure creation. Bulk viscosity and matter creation generate cosmological models with same physical properties , but the identity between the two process is not generically valid. Cosmic dynamics generated by each can be mimicked by the other one. However, the thermodynamic features are quite different [8].
We obtain the equation for the evolution of the density contrast and the respective modes for OSC. Two different situations are considered: first, we consider that the produced particles affect the formation of the inhomogeneities; second, the creation of particles will be considered smooth, consequently do not participate of the inhomogeneities production. We defend, in this work, that a coherent model for the universe must provide a growing mode for the density contrast. Eventhough, we lived in an accelerated universe.

Using this criterious, we can establish if the process of the cosmic particle creation is a homogeneous process or not.

\section{OPEN SYSTEM THERMODYNAMICS}

In the presence of cosmic matter creation, the appropriate analysis is performed in the context of thermodynamics of open systems [9], that results in a reinterpretation of the stress energy tensor. Considering adiabatic transformations, the thermodynamical energy conservation, with a source of particles, can be written as [9]

$$
d(\varepsilon V)+P_{t h} d V-\frac{E d(n V)}{n}=0,
$$

where $n$ is the particle density, $E=\varepsilon+P_{t h}$ is the enthalpy per unit volume, $P_{t h}$ is the thermodynamical pressure and $\varepsilon$ is the energy density. In such a transformation, the 'heat' received by the system is due entirely to the change of the number of particles. In the cosmological context, the particle production is due to the transfer of energy from gravitational field. Hence, the creation of matter acts as source of internal energy.

We consider the cosmic medium describe by the energy momentum tensor

$$
T^{\alpha \beta}=\varepsilon u^{\alpha} u^{\beta}+P h^{\alpha \beta},
$$

where $h^{\alpha \beta}$ is the projection tensor and $u^{\alpha}$ is the four-velocity vector. The $P$ is the total pressure that includes the thermodynamical pressure and an additional pressure related to the creation process [9], namely,

$$
P=P_{t h}+\tilde{P}
$$


The creation process will be considered adiabatic, which here means that the entropy per particle is constant. Under this condition, the equilibrium entropy per particle does not change as it does in dissipative process.

The Einstein's field equations imply the equations of motion,

$$
h_{\beta}^{\alpha} T^{\gamma \beta}=0
$$

and the energy balance

$$
u^{\alpha} T_{; \alpha}^{\alpha \beta}=0
$$

Additionally, according to the second law of thermodynamics $\left(S_{; \alpha}^{\alpha} \geq 0\right)$, the only particle number variations admitted are such that we have particle creation, governing by

$$
\dot{n}+n \theta=\Psi
$$

where $\Psi$ is the source of particles and $\theta$ is the universe expansion. tion

Using the thermodynamic second law and the Euler equa-

$$
\mu=\frac{\varepsilon+P_{t h}}{n}-T \sigma,
$$

we can write the pressure creation as [10]

$$
\tilde{P}=-\frac{\left(\varepsilon+P_{t h}\right) \Psi}{n \theta}
$$

where $\mu$ is the chemical potential and $\sigma$ is the entropy per particle.

The zeroth order background will be governed by the line element

$$
d s^{2}=-d t^{2}+R(t)^{2} \gamma_{a b} d x^{a} d x^{b}
$$

where $\gamma_{a b}$ is the 3 - space metric. Consequently, Einstein's field equations reduce to

$$
\begin{array}{r}
3\left(\frac{\dot{R}}{R}\right)^{2}=8 \pi G \varepsilon, \\
2\left(\frac{\dot{R}}{R}\right)^{\cdot}=-8 \pi G(\varepsilon+P),
\end{array}
$$

where the dot denotes the time derivative.

Using the metric (9), the energy balance equation (5) becomes

$$
\dot{\varepsilon}=-3 \frac{\dot{R}}{R}(\varepsilon+P) \text {. }
$$

Taking into account the particle number density and the temperature as are our basic variables we can infer the state equations

$$
\begin{gathered}
P_{t h}=P_{t h}(n, T), \\
\varepsilon=\varepsilon(n, T), \\
\sigma=\sigma(n, T) .
\end{gathered}
$$

Making use of Gibbs relation

$$
n T d \sigma=d \varepsilon-\frac{\varepsilon+P_{t h}}{n} d n
$$

and equation (14), a general relation can be written, namely

$$
\frac{\partial \varepsilon}{\partial n}=\frac{\varepsilon+P_{t h}}{n}-\frac{T}{n} \frac{\partial P_{t h}}{\partial T} .
$$

Equation for evolution of the temperature is calculated considering relations (12) and (17), resulting

$$
\dot{T}=\left(\frac{\partial \varepsilon}{\partial T}\right)^{-1}\left\{3 \frac{\dot{R}}{R}\left(\tilde{P}+\frac{\partial P_{t h}}{\partial T} T\right)+\Psi\left(\frac{\varepsilon+P_{t h}}{n}-\frac{T}{n} \frac{\partial P_{t h}}{\partial T}\right)\right\}
$$

The sound velocity in the cosmic fluid is defined by

$$
v_{s}^{2}=\frac{\partial P_{t h}}{\partial \varepsilon},
$$

that can be reformulated using equation (17), assuming the form:

$$
v_{s}^{2}=\frac{n}{\varepsilon+P_{t h}} \frac{\partial P_{t h}}{\partial n}+\frac{T\left[\frac{\partial P_{t h}}{\partial T}\right]^{2}}{\left(\varepsilon+P_{t h}\right) \frac{\partial \varepsilon}{\partial T}} .
$$

Taking into account the relation

$$
\dot{P}_{t h}=\frac{\partial P_{t h}}{\partial n} \dot{n}+\frac{\partial P_{t h}}{\partial T} \dot{T}
$$

and equations (6), (18) and (19), evolution of the pressure reads

$$
\begin{aligned}
& \dot{P_{t h}}=-3 \frac{\dot{R}}{R}\left(\varepsilon+P_{t h}\right)\left\{v_{s}^{2}+\frac{\frac{\partial P_{t h}}{\partial T}}{\frac{\partial \varepsilon}{\partial T}} \frac{\tilde{P}}{\varepsilon+P_{t h}}\right\} \frac{\Psi}{n}\left(\varepsilon+P_{t h}\right) \\
& \left\{v_{s}^{2}-\frac{\frac{\partial P_{t h}}{\partial T}}{\frac{\partial \varepsilon}{\partial T}}\right\} .
\end{aligned}
$$

Finally, the time evolution of the particle entropy density can be found using Gibbs relation and equations (6) and (18),namely

$$
n T \dot{\sigma}=\frac{\varepsilon+P_{t h}}{n}\left\{3 n \frac{\dot{R}}{R} z-\Psi\right\},
$$

where

$$
z=-\frac{\tilde{P}}{\varepsilon+P_{t h}}=\frac{\dot{N}}{N \theta} .
$$

We sum up this section writing the time evolution of the particle density, temperature and pressure in the adiabatic case. It is done using (6), (18) and (22)

$$
\begin{gathered}
\dot{n}=-3 \frac{\dot{R}}{R}(1-z), \\
\dot{T}==3 \frac{\dot{R}}{R} T\left(\frac{\frac{\partial P_{t h}}{\partial T}}{\frac{\partial \varepsilon}{\partial T}}\right), \\
\dot{P_{t h}}=-3 \frac{\dot{R}}{R}(\varepsilon+P) v_{s}^{2} .
\end{gathered}
$$




\section{THE PERTURBED EQUATIONS}

The metric perturbations are defined by

$$
\begin{array}{r}
\delta g_{a b}=A \delta_{a b}+B_{, a b}, \\
\delta g_{a 4}=F_{, 4}, \\
\delta g_{44}=E,
\end{array}
$$

where the quantities $A, B, F$ and $E$ are space and time dependent scalars.

From normalization condition, $u_{\mu} u^{\mu}=1$, the four velocity in first order is given by

$$
\begin{array}{r}
\hat{u_{4}}=\hat{u^{4}}=\frac{R^{2} E}{2}, \\
R^{2} \hat{u^{a}}+R^{2} F_{, a}=\hat{u_{a}} \equiv v_{, a},
\end{array}
$$

where the hat indicates a perturbed quantity.

Perturbing to first order the equation for the conservation of particle density $\left(n u^{\alpha}\right)_{; \alpha}=\Psi$, results

$$
\left(\frac{\hat{n}}{n}\right)^{\cdot}+R^{-2} \nabla v-\nabla F+\frac{\dot{f}}{2}=\frac{\hat{\Psi}}{n}-\frac{\Psi}{n}\left(\frac{\hat{n}}{n}+\frac{R^{2} E}{2}\right) .
$$

The energy balance and momentum balance equations to first order are respectively:

$$
\begin{aligned}
\dot{\hat{\varepsilon}}+3 \frac{\dot{R}}{R}(\hat{\varepsilon}+\hat{P})+\left(R^{-2} \nabla v+\frac{\dot{f}}{2}-\nabla F\right)(\varepsilon+P) & =0 \\
\hat{P}_{, \mu}+\dot{P} v_{, \mu}+(\varepsilon+P) v_{, \mu}-R^{2} \frac{\varepsilon+P}{2} E_{, \mu} & =0 .
\end{aligned}
$$

To complete the set of basic equations we need of the linearized field equations [7]:

$$
\begin{gathered}
\hat{G}_{4}^{4}=R^{-2} \nabla A-\frac{\dot{R}}{R} \dot{f}+2 \frac{\dot{R}}{R} \nabla F-3 \dot{R}^{2} E=-\hat{\varepsilon}, \\
\hat{G}_{4 \alpha}=-\dot{A}_{, \alpha}-R \dot{R} E_{, \alpha}-\left(\dot{R}^{2}+2 R \ddot{R}\right) F_{, \alpha} \\
=-(\varepsilon+P) v_{, \alpha}+R^{2} P F_{, \alpha} .
\end{gathered}
$$

\section{DENSITY CONTRAST EVOLUTION EQUATION}

The perturbed variables, introduced in the preceding section, change their values under the change of correspondence between the perturbed world and unperturbed background. The change of the correspondence is formally expressed in terms of a coordinate transformation in the perturbed world, which is called a gauge transformation. In the linear perturbation theory it is necessary only to consider infinitesimal gauge transformation

$$
x^{\prime \alpha}=x^{\alpha}-\xi^{\alpha} .
$$

W. Zimdahl shows in his work [5] how to describe cosmological perturbations thermodynamically oriented by suitable quantities which are invariant under transformations (38), with an obvious physical meaning and obey reasonable equations. by

The behavior of scalars under transformation (38) are given

$$
S^{\prime}(x)-S(x)=\xi^{n} S_{, n} .
$$

Therefore, the scalar quantities of the preceding section transform as

$$
\begin{array}{r}
A^{\prime}=A+2 \frac{\dot{R}^{2}}{R} \xi^{0}, \\
B^{\prime}=B+2 R^{-2} \xi, \\
F^{\prime}=F+R^{-2} \xi^{0}+\left(R^{-2} \xi\right)_{, 0}, \\
E^{\prime}=E-2 R^{-2} \xi_{0}^{0}, \\
v^{\prime}=v-\xi^{0}, \\
\hat{n}^{\prime}=\hat{n}-3 \frac{\dot{R}}{R} n \xi^{0}(1-z), \\
\hat{T}^{\prime}=\hat{T}-3 \frac{\dot{R}}{R} n \xi^{0} T\left(\frac{\frac{\partial P_{t h}}{\partial T}}{\frac{\partial \varepsilon}{\partial T}}\right)(1-z) .
\end{array}
$$

The basic gauge invariant variables that we shall take are defined by

$$
s=\frac{\hat{n}}{n}-3 \frac{\dot{R}}{R} v(1-z)
$$

and

$$
r=\frac{\hat{T}}{T}-3 \frac{\dot{R}}{R} v \frac{\frac{\partial P_{t h}}{\partial T}}{\frac{\partial \varepsilon}{\partial T}}(1-z)
$$

On the other hand,a gauge invariant gravitational field variable from the point of view of the matter [6] is:

$$
q=\frac{3}{2} A+3 \frac{\dot{R}}{R} v
$$

The gauge invariant description of the system of the perturbed equations in the last section can be put in terms of the variables $q, r$ and $s$. So, the energy density and pressure perturbations are gauge invariantly characterized by:

$$
\begin{array}{r}
\hat{\varepsilon}_{c}=\hat{\varepsilon}-3 \frac{\dot{R}}{R} v(\varepsilon+\tilde{P}), \\
\hat{P}_{c}=\hat{P}_{t h}-3 \frac{\dot{R}}{R} v(\varepsilon+\tilde{P}) v_{s}^{2} .
\end{array}
$$

Using equation (47), equation (50) assumes the form

$$
\hat{\varepsilon}_{c}=\left(\varepsilon+P_{t h}\right) s+T a,
$$

where

$$
a=\frac{\partial \varepsilon}{\partial T} r-\frac{\partial P_{t h}}{\partial T} s
$$


Introducing the gauge invariant variables, the particle number balance (33) may be written as

$$
\begin{aligned}
& \dot{s}+(1-z) \dot{q}+\frac{3}{2} \gamma \frac{\dot{R}}{R} \frac{\hat{\varepsilon}_{c}}{\varepsilon+P_{t h}}-\frac{k^{2}}{3 \dot{R} R} q \\
& +9 \frac{\dot{R}^{2}}{R^{2}} z(1-z) v\left(1+\frac{\gamma}{2}\right)-3 \frac{\dot{R}}{R} v \dot{z}+3 \frac{\dot{R}}{R} z s=\frac{\hat{\Psi}}{n},
\end{aligned}
$$

where $\gamma=1+\frac{P_{t h}}{\varepsilon}$. To find the last result it is necessary to use the perturbed field equations (36), (37), the field equation

$$
\left(\frac{\dot{R}}{R}\right)^{\cdot}=-\frac{3}{2} H^{2} \gamma(1-z)
$$

and assumes the spatial dependence $e^{\mathbf{i k} \cdot \mathbf{r}}$ with constant comoving wavector $k$ for all first-order quantities.

With auxilious of equation (54) we can change the form of the energy and pressure balance equations, given by, respectively

$$
\frac{\left(a R^{3}\right)^{\cdot} T R^{-3}}{\varepsilon+P_{t h}}+\frac{z k^{2} q}{3 R \dot{R}}+\frac{\hat{\psi}}{n}=-3 H z \frac{\hat{P_{t h}}-\frac{\gamma}{2} \hat{\varepsilon}}{\varepsilon+P_{t h}}-\frac{3 H \hat{\tilde{P}}}{\varepsilon+P_{t h}}
$$

and

$$
\begin{gathered}
s v_{s}^{2}-v \dot{z}+3 H z(1-z) v\left(1+v_{s}^{2}\right)+\frac{\hat{\tilde{P}}}{\varepsilon+P_{t h}} \\
+\quad \frac{R}{3 \dot{R}} \dot{q}(1-z)+T \frac{\frac{\partial P_{t h}}{\partial T}}{\frac{\partial \varepsilon}{\partial T}} \frac{a}{\varepsilon+P_{t h}}=0
\end{gathered}
$$

Substituting equation (56) into (54) we obtain

$$
\begin{aligned}
& \dot{s}-3 H s v_{s}^{2}+9 H^{2} z(1-z) v\left(\frac{\gamma}{2}-v_{s}^{2}\right)-\frac{3 H \hat{\tilde{P}}}{\varepsilon+P_{t h}} \\
& -\frac{3 H T}{\varepsilon+P_{t h}} a \frac{\frac{\partial P_{t h}}{\partial T}}{\frac{\partial \varepsilon}{\partial T}}+\frac{3}{2} \gamma H \frac{\hat{\varepsilon_{c}}}{\varepsilon+P_{t h}}-\frac{k^{2} q}{3 R \dot{R}}+3 H z s=\frac{\hat{\Psi}}{n} .
\end{aligned}
$$

Using eqs. (54) and (53), results

$$
\begin{aligned}
& \dot{s}-3 H \frac{1-z}{\varepsilon+P_{t h}}\left(\hat{P}_{c}-\frac{\gamma}{2} \hat{\varepsilon}_{c}\right)-(1-z) \frac{k^{2} q}{3 R \dot{R}} \\
& +3 H z s=-\frac{T}{R^{3}}\left(R^{3} a\right)
\end{aligned}
$$

where the evolution equation for $s$ is put in terms of the gauge invariant variables, only.

We focus our attention on the adiabatic case, that is equivalent to consider $a=0$ [5]. So, equation (59) assumes the form

$$
\dot{s}-3 H(1-z)\left(v_{s}^{2}-\frac{\gamma}{2}\right) s-(1-z) \frac{k^{2} q}{3 R \dot{R}}+3 H z s=0,
$$

where the variable $s$ is rewritten as

$$
s=\frac{\hat{\varepsilon}}{\varepsilon+P_{t h}} .
$$

A manipulation of equation (59) results

$$
\begin{aligned}
& \ddot{s}+\left\{-\frac{3}{2}\left\{H(1-z)\left(2 v_{s}^{2}-\gamma\right)\right\}+3 H z+\left(\frac{\ddot{R}}{\dot{R}}\right.\right. \\
& \left.\left.+\frac{\dot{R}}{R}\right)+\frac{\dot{z}}{1-z}\right\} \dot{s}+\left\{-\frac{3}{2}\left\{H(1-z)\left(2 v_{s}^{2}-\gamma\right)\right\}+3(H z)\right. \\
& +\left\{3 \frac{H z}{1-z}-\frac{3}{2} H\left(2 v_{s}^{2}-\gamma\right)\right\}\left\{(1-z)\left(\frac{\ddot{R}}{\dot{R}}+\frac{\dot{R}}{R}\right)+\dot{z}\right\} \\
& \left.+\frac{k^{2}}{R^{2}} v_{s}^{2}\right\} s-\frac{k^{2}}{R^{2}} v \dot{z}=0 .
\end{aligned}
$$

The equation (62) furnish the dynamics of the adiabatic perturbations in a universe with particle production, where the particles are formed at the expenses of the gravitational field. For a universe with null pressure small sound velocity in the cosmic medium and the absence of the creation process, equation (62) becomes the usual density contrast equation for the standard model [4]:

$$
\ddot{s}+2 H \dot{s}-4 \pi G \varepsilon s=0 .
$$

\section{IS THE PARTICLE PRODUCTION HOMOGENEOUS?}

The particle production in the universe is a global homogeneous process or not? We want to give a answer for this question considering the large scale structure formation and the recent results of the observation of the supernovas of the type IA, that indicates an accelerated universe.

Taking into account that the structures existing in the universe are formed from small perturbations in the past, more specifically scalar perturbations, a coherent cosmological model, with this assumption, must produce a growing mode for the density contrast. Apart from this, the mode must grows even in an accelerated scenario for the universe.

In order to measure the effects of particle production in the expansion of the universe, Lima et al. [8], introduce the dimensionless parameter $\beta=\frac{\Psi}{3 n H}$.

Combining eqs. (8), (9) and (10), we can write the field equation

$$
R \ddot{R}+\Delta(\dot{R})^{2}=0,
$$

where $\Delta=\frac{3}{2} \gamma(1-\beta)-1$. Integrating (64), the scale factor resulting is

$$
R=R_{0}\left(\frac{t}{t_{0}}\right)^{\frac{1}{1+\Delta}}
$$

where the subscript 0 alludes to the present time.

The deceleration parameter, $q=-\frac{\ddot{R} R}{(\dot{R})^{2}}$, for the universe expanding conform the scale factor (5.2) is given by $q=$ $-1+\frac{3}{2} \gamma(1-\beta)$. Consequently, a dust universe will be accelerated for $\beta>\frac{1}{3}$.

Substituting the scale factor (65) in eq. (62), considering $v_{s}<<1$ and using long wavelength limit approximation, we obtain

$$
\ddot{s}+C_{1} \dot{s}+C_{0} s=0,
$$


where

$$
C_{1}=\frac{2(3 \beta+2)}{3 \gamma(1-\beta) t}
$$

and

$$
C_{0}=-\frac{-2}{t^{2}}-\frac{4}{3 \gamma(\beta-1)^{2} t^{2}}\left\{3 \beta^{2}-1+4 \beta-\frac{4 \beta}{\gamma}\right\} .
$$

Integrating the eq. (66) we obtain the modes

$$
\begin{gathered}
s_{-}(t)=C_{1} t^{-1+\frac{2 \beta}{\gamma(\beta-1)},} \\
s_{+}(t)=C_{2} t^{\frac{4}{3 \gamma(\beta-1)}+2},
\end{gathered}
$$

where the usual modes of the standard model are obtained for $\beta=0$.

In accord with the second law of thermodynamics $\left(S_{; \alpha}^{\alpha} \geq 0\right)$, only particle production process is possible. Consequently, $\beta \geq 0$. On the other hand, for $\beta>1$ the dominant energy condition is broken. So, in principle, the validity range for $\beta$ parameter is $0 \leq \beta \leq 1$, and $\frac{1}{3} \leq \beta \leq 1$ assuming that the universe expansion is accelerated

Although the decreasing mode can be important in some circumstances, we shall hereafter mainly deal only with the increasing mode. It is responsible for the formation of cosmic structure in the gravitational stability picture. Taking into account the decreasing mode, the universe would not have been homogeneous in the past. Besides, for a growing mode that starts to grow just after the end of radiation era, Peebles argued that the contribution of decaying solution must be negligible [18].

The equation (69) is an increasing mode for $\beta<-1$, but, considering the validity range for $\beta$, the mode (69) is a decaying mode. Otherwise, the mode (70) is a growing mode for $\beta<\frac{1}{3}$. Resuming, we not have a growing mode for the density contrast in a acceptable range for the creation parameter $\beta$.

To obtain equation (62), we perturb the source of particles. Therefore, the creation process is inhomogeneous, in the sense that the created particles contributes to the inhomogeneities formation in the universe.

On the other hand, if we consider that the cosmic particle production do not participate of the inhomogeneities formation, the particle production mimics, in this sense, the inclusion of a smooth $\Lambda$ term. Consequently, with null contribution to the inhomogeneities birth. In this scenario, where the source of particles is not perturbed, the equation for the density contrast is given by

$$
\ddot{s}+2 H \dot{s}-4 \pi G \varepsilon s(1+3 \gamma)(1+\gamma) s=\gamma \frac{\nabla^{2} s}{R^{2}(\beta-1)} .
$$

Taking into account the long wavelength limit approximation and the scale factor (65), the integration of eq. (71) furnishes

where $A=\sqrt{9 \beta^{2}+25+6 \beta}$

$$
\begin{aligned}
& s_{a}(t)=t^{\frac{3 \beta+1+A}{6 \beta-6}}, \\
& s_{b}(t)=t^{\frac{3 \beta+1-A}{6 \beta-6}},
\end{aligned}
$$

The mode $s_{a}$ is a decaying mode and the mode $s_{b}$ is a growing mode, for $0 \leq \beta \leq 1$. For $\beta=0$ the usual solutions for the growing and decaying modes of the standard model are obtained.

In conclusion, we have an growing mode for the density contrast in an accelerated phase for the universe described by the OSC model, if the creation process is homogeneous.

Another possibility, is to admit that the era when the small deviations from the homogeneity occurs, the expansion of the universe is not accelerated.

Naturally, if the conclusions are dependent of the source of the particles considered, this is a question for a future investigation.

\section{Acknowledgements}

The author is thankful to the organizing committee of the 100 years of relativity conference at São Paulo, Brazil, and to the brazilian agency, CNPQ, for the financial support.
[1] V. F. Mukhanov, H. A. Feldman and R. H. Brandenberger, Phys. Rep., 215 , 203-333 (1992).

[2] J. M. Bardeen, Phys. Rev., D22 , 1882 (1980).

[3] G. R. F. Ellis and M. Bruni. Phys. Rev., D53, 4287 (1989).

[4] T. Padmanabhan, Structure formation in the universe , (Cambridge University Press, Cambridge, 1993) .

[5] W. Zimdhal, D. Pavon and D. Jou Class.Quantum Grav., 8, 677,(1993).

[6] V. N. Lukash. Sov. Phys-JEPT Lett., 12, 307,(1980).

[7] H. Kodama and M. Sazaki Prog. Theor. Phys. Suppl, 78, 1, (1984) .

[8] J. A. Lima and A. S. Germano. Phys.Lett, 170A, 373,(1992)

[9] I. Prigogine, J. Geheniau, E. Gunzig and P. Nardone. Gen. Relativ. Grav., 21, 8, 767,(1989).

[10] M. O. Calvão, J. A. S. Lima and I. Waga. Phys.Lett, 162A,
223,(1992)

[11] J. A. S. Lima, A. S. M. Germano and L. R. W. Abramo. Phys. Rev., D53 , 4287 (1996).

[12] S.Weinberg. Gravitation and Cosmology Principles and Applications of the General Theoryof Relativity (Jonh Wiley \& Sons, New York, 1972).

[13] E. W. Kolb and M. S. Turner. The EarlyUniverse (AddisonWesley ,Redwood City,California,1990).

[14] S. Pelmuter et al. preprint astro-ph/9812473.

[15] A. G. Riess, et al., Astrophys. J. 116,1009 (1998).

[16] R. M. Wald. General Relativity (University of Chicago Press, Chicago, 1992).

[17] W. Zimdahl, A. B. Balakin and D. Pavon astro-ph/0009353

[18] J. Peebles, et al. Astrophys. J, 147, 859, (1967). 